\title{
Schizophrenia polygenic risk scores in youth mental health: preliminary associations with diagnosis, clinical stage and functioning
}

Jacob J. Crouse, Joanne S. Carpenter, Frank Iorfino, Tian Lin, Nicholas Ho, Enda M. Byrne, Anjali K. Henders, Leanne Wallace, Daniel F. Hermens, Elizabeth M. Scott, Naomi R. Wray and lan B. Hickie

\section{Background}

The schizophrenia polygenic risk score (SCZ-PRS) is an emerging tool in psychiatry.

\section{Aims}

We aimed to evaluate the utility of SCZ-PRS in a young, transdiagnostic, clinical cohort.

\section{Method}

SCZ-PRSs were calculated for young people who presented to early-intervention youth mental health clinics, including 158 patients of European ancestry, 113 of whom had longitudinal outcome data. We examined associations between SCZ-PRS and diagnosis, clinical stage and functioning at initial assessment, and new-onset psychotic disorder, clinical stage transition and functional course over time in contact with services.

\section{Results}

Compared with a control group, patients had elevated PRSs for schizophrenia, bipolar disorder and depression, but not for any non-psychiatric phenotype (for example cardiovascular disease) Higher SCZ-PRSs were elevated in participants with psychotic, bipolar, depressive, anxiety and other disorders. At initial assessment, overall SCZ-PRSs were associated with psychotic disorder (odds ratio (OR) per s.d. increase in SCZ-PRS was 1.68, $95 \% \mathrm{Cl} 1.08-2.59, P=0.020)$, but not assignment as clinical stage $2+$ (i.e. discrete, persistent or recurrent disorder) $(\mathrm{OR}=0.90$,
95\% $\mathrm{Cl} 0.64-1.26, P=0.53)$ or functioning $(R=0.03, P=0.76)$. Longitudinally, overall SCZ-PRSS were not significantly associated with new-onset psychotic disorder $(\mathrm{OR}=0.84,95 \% \mathrm{Cl}$ $0.34-2.03, P=0.69)$, clinical stage transition $(\mathrm{OR}=1.02,95 \% \mathrm{Cl}$ $0.70-1.48, P=0.92)$ or persistent functional impairment (OR = $0.84,95 \% \mathrm{Cl} 0.52-1.38, P=0.50$ ).

\section{Conclusions}

In this preliminary study, SCZ-PRSs were associated with psychotic disorder at initial assessment in a young, transdiagnostic, clinical cohort accessing early-intervention services. Larger clinical studies are needed to further evaluate the clinical utility of SCZ-PRSS, especially among individuals with high SCZPRS burden.

\section{Keywords}

Genetics; youth mental health; psychiatry; early intervention; transdiagnostic.

\section{Copyright and usage}

(C) The Author(s), 2021. Published by Cambridge University Press on behalf of the Royal College of Psychiatrists. This is an Open Access article, distributed under the terms of the Creative Commons Attribution licence (http://creativecommons.org/ licenses/by/4.0/), which permits unrestricted re-use, distribution, and reproduction in any medium, provided the original work is properly cited.

\section{Major developments in psychiatric genetics}

The past decade has witnessed two major conceptual shifts in our understanding of the genetic architecture of mental disorders. First, in contrast to disorders caused by a single genetic variant (such as huntingtin in Huntington's disease ${ }^{1}$ ), genetic risk for mental disorders involves the contribution of hundreds or thousands of common variants of small effect ${ }^{2}$ and/or rare variants of larger effect (for example duplications, deletions). ${ }^{3}$ That is, they are 'polygenic'. ${ }^{4}$ Notably, even classic monogenic disorders such as Huntington's disease ${ }^{5}$ and $B R C A 1$ breast cancer ${ }^{6}$ are recognised to have a polygenic component influencing age at onset.

Strong evidence of polygenicity in mental disorders has come from international collaborative psychiatric genome-wide association studies (GWASs), ${ }^{2,7-10}$ For example, major efforts by the Psychiatric Genomics Consortium (PGC) have identified 108 genetic loci associated with schizophrenia, ${ }^{2} 102$ loci associated with major depression ${ }^{11}$ and 30 loci associated with bipolar disorder. $^{8}$ A key implication of these studies is that the causal impact of single variants is likely to be small, and vulnerability for complex psychiatric phenotypes is associated with a high load of risk variants.

The second major conceptual shift is that the genetic architec ture of the major mental disorder diagnoses overlaps across disorders. For example, the Cross-Disorder group of the PGC has provided direct molecular evidence of shared genetic risk, reporting high genetic correlations (based on single-nucleotide polymorphisms (SNPs)) between schizophrenia and bipolar disorder; and moderate genetic correlations between schizophrenia and depression; bipolar disorder and depression; and attention-deficit hyperactivity disorder (ADHD) and depression. ${ }^{12}$

A recent study of GWAS data on 25 common brain disorders supported the high degree of shared genetic risk among major mental disorders (ADHD, depression, bipolar disorder, schizophrenia), whereas neurological disorders such as generalised epilepsy, Alzheimer's disease, Parkinson's disease and multiple sclerosis were more genetically distinct, suggesting greater diagnostic specificity and/or distinct aetiologies for neurological disorders. ${ }^{13}$ These and other studies ${ }^{14,15}$ suggest that alterations in key biological pathways (for example neuronal, immune) are frequently shared across the major mental disorders, particularly during brain development.

\section{Polygenic risk scores in psychiatry}

A recent tool emerging from psychiatric GWASs that captures features of these two conceptual shifts is the 'polygenic risk score' (PRS) ${ }^{16}$ A PRS is an estimate of an individual's genetic liability to a particular trait or phenotype, calculated as a weighted count of risk alleles, with the risk alleles and their weights derived from GWASs (noting that these individuals are unrelated to the samples included in the GWAS). ${ }^{17}$ Although the effect sizes of known variants are currently too small for outcome prediction 
using any single variant, the PRS framework allows incorporation of many variants of small effect (and is robust to inclusion of false positives) to produce an aggregate index of liability to disorder.

Although the variance in liability to schizophrenia explained by the PRS (SCZ-PRS) is only $\sim 7 \%,{ }^{2}$ several studies have examined the potential clinical utility of the SCZ-PRS. Several studies to date have shown that in samples with psychotic disorders, the SCZ-PRS is strongly and robustly associated with the diagnosis of schizophrenia, ${ }^{18}$ transition from clinical high risk or a different mental disorder (for example depression) to full-threshold psychotic disorder, ${ }^{19-21}$ poorer neurocognition ${ }^{22,23}$ and social cognition, ${ }^{22}$ negative symptoms $^{24}$ and poorer illness course; ${ }^{23,25}$ however, it is important to note that some studies have not observed significant associations between the SCZ-PRS and aspects of illness course (such as treatment resistance), ${ }^{26,27}$ and impairments in overall neurocognition ${ }^{28}$ and specific neurocognitive domains. ${ }^{29,30}$ Finally, the SCZ-PRS has been observed to be positively associated with other mental disorders including depression, bipolar disorders, substance use disorders and anxiety disorders, among others (i.e. 'genetic pleiotropy'). ${ }^{18,31}$

\section{Current study}

The shared genetic risk across major mental disorders and pleiotropy of the SCZ-PRS begs the question of whether it could have utility for predicting outcomes in broader transdiagnostic samples. ${ }^{32}$ There has been a shift toward a recognition of transdiagnostic models of mental disorders that acknowledge the dynamic nature of syndrome-based phenotypes $^{33}$ and their limited specificity to aetiology, genetic architecture, risk factors and neurobiology. ${ }^{13,34-39}$ Efforts to improve prediction of illness trajectories and outcomes is particularly important in young people in the early phases of mental disorders, during which syndromes and diagnoses are more plastic. ${ }^{33,40-42}$ Accordingly, this study aimed to evaluate the utility of PRSs in a transdiagnostic clinical cohort of adolescents and young adults accessing early-intervention mental health services. While PRSs were calculated for a range of psychiatric (for example depression, bipolar disorder) and non-psychiatric phenotypes (for example body mass index, peptic ulcer disease, type 2 diabetes, cardiovascular disease), the current study focuses on the schizophrenia PRS. Specifically, we aimed to examine associations between SCZ-PRS and diagnosis, clinical stage and functioning around the time of entry to clinical services, and between SCZ-PRS and new-onset psychotic disorder, clinical stage transition and persistent functional impairment over time in contact with clinical services.

\section{Method}

\section{Human ethics and study reporting}

The authors assert that all procedures contributing to this work comply with the ethical standards of the relevant national and institutional committees on human experimentation and with the Helsinki Declaration of 1975, as revised in 2008. All procedures involving human patients were approved by the University of Sydney Human Research Ethics Committee (2012/1626, 2012/ 1631). Written informed consent was obtained from all patients and/or their guardians. This study followed the Strengthening the Reporting of Observational Studies in Epidemiology (STROBE) reporting guideline. ${ }^{43}$

\section{Participants}

Study participants were drawn from a large research case register of consecutive referrals to youth mental health clinics at the Brain and Mind Centre in Sydney, Australia between 2004 and 2018, and were recruited to a neurobiological study of the early phases of mental disorders. ${ }^{44}$ These clinics (such as 'headspace') provide highly accessible and youth-friendly early-intervention services for young people experiencing problems with substance use and/or mental health, attracting young people with a range of subthreshold and full-threshold mental health syndromes (commonly mood, anxiety and psychotic syndromes). ${ }^{44}$ headspace consists of an integrated mix of primary-level and specialist services, and participants were receiving clinician-based case management and relevant evidence-based social, psychological and/or medical interventions as part of standard clinical care, which may have involved contact with a psychiatrist, psychologist, occupational therapist, social worker or hospital admission. ${ }^{44}$

\section{Eligibility criteria}

Eligibility criteria for this study were:

(a) an available SCZ-PRS;

(b) aged $12-30$ at baseline;

(c) European ancestry; and

(d) willing/able to give informed consent (and/or parental consent was obtained).

Potential participants were excluded from the broader neurobiological study (and by extension this study) if they had:

(a) history of neurological disease;

(b) medical illness known to affect brain function (such as epilepsy);

(c) received electroconvulsive therapy in the 3 months prior to assessment;

(d) clinically determined intellectual disability (i.e. IQ < 70); and/or

(e) insufficient understanding of the English language to allow participation in verbal assessments/testing.

\section{Clinical and functional outcomes}

The methodology used here is described in greater detail elsewhere. ${ }^{44-47}$ Briefly, trained research staff used a standardised clinical proforma to gather demographic, clinical and functioning data from research and clinical case files across eight predetermined time points. The proforma collects standardised information regarding:

(a) basic demographics (such as gender, age);

(b) subthreshold and full-threshold mental health diagnoses;

(c) clinical course (such as clinical stage, admission to hospital);

(d) comorbidities (such as physical health conditions); and

(e) functioning.

Phase I and II of data extraction of the 'Optymise' cohort concluded in 2019, and the cohort comprises 2901 participants from our clinical case register. ${ }^{44}$ In the current study, we focused on the following outcomes: mental disorder diagnoses, clinical stage and functioning.

\section{Mental disorder diagnoses}

Mental disorder diagnoses were classified according to DSM-5 criteria $^{48}$ and labelled as either primary, secondary or tertiary based on judgement of which was the dominant presenting problem at the particular time point. Diagnosis was determined solely by diagnosis reported and recorded by the treating clinician(s) as presented in clinical notes or symptomatology. Based on information recorded in the clinical notes, researchers determined whether DSM-5 criteria were met for a disorder at that time point. If symptomatology recorded in the notes indicated some, but not all criteria being met for a disorder, then a subthreshold classification was recorded. 


\section{Clinical stage}

Clinical stage was assigned according to an established model. ${ }^{33,49,50}$ Descriptions of the criteria for the stages within this model are detailed elsewhere, ${ }^{49}$ and a decision tree is available in ${ }^{44}$. Briefly, individuals are assigned to one of six stages including: stage 0 (no current symptoms; increased risk of disorder); stage 1a (mild or non-specific symptoms); stage $1 \mathrm{~b}$ (moderate but subthreshold symptoms); stage 2 (full-threshold disorder with moderate to severe symptoms); stage 3 (incomplete remission or relapse); or stage 4 (severe, unremitting or refractory illness). ${ }^{33}$

\section{Functioning}

Functioning was measured by the clinician-rated Social and Occupational Functioning Assessment Scale (SOFAS). ${ }^{51}$ The SOFAS is a 100-point scale (higher scores denoting better functioning), with instructions to raters to avoid confounding the rating of functioning with symptoms. A SOFAS score of below 70 is considered to indicate clinically significant impairment. ${ }^{52}$

\section{PRSS}

A subset $(n=193)$ of the cohort had blood collected and genotyped at the Queensland Institute for Medical Research Berghofer Molecular Epidemiology Laboratory using the Illumina Psych Chip v1.0 under standard protocols. Stringent quality control procedures were implemented in GenomeStudio and Plink2 and applied to these data plus an independently collected control sample. This independent control sample $(n=1528)$ comprised unaffected Australians who were genotyped in case-control studies of motor neuron disease and Parkinson's disease (details available from the authors on request). ${ }^{53}$ Briefly, SNPs were filtered for call missingness $>10 \%$, departure from Hardy-Weinberg equilibrium $\left(P<10^{-6}\right)$, minor allele frequency $<0.01$, and deviation from allele frequency compared with the Haplotype Reference Consortium. ${ }^{54}$ After quality control, genotyped SNPs were submitted to the Sanger Imputation Server for imputation to the Haplotype Reference Consortium reference samples. Using the PC projection method, the samples were projected to the 1000 Genome reference samples, ${ }^{55}$ and then assigned to a population if they clustered with the population within 3 s.d.s.

Among the 193 clinical samples, 161, 10 and 22 were assigned European, East Asian and other ancestry, respectively, based on genetic data. Here, we focus on participants of European ancestry. PRSs were generated for eight traits using SBayesR: ${ }^{56}$ three mental disorders/traits (schizophrenia, ${ }^{57}$ bipolar disorder, ${ }^{8}$ depression ${ }^{11}$ ), height, ${ }^{58}$ and four traits of relevance to common comorbidities of mental disorders (body mass index, ${ }^{58}$ cardiovascular disease, ${ }^{59}$ type 2 diabetes, ${ }^{59}$ peptic ulcer disease ${ }^{60}$ ). The PRSs of the control and clinical participants were standardized by subtracting the mean and dividing by the s.d. of the control sample. Here, we focus on the SCZ-PRS based on it having the largest GWAS discovery sample in psychiatry, ${ }^{2}$ and to limit the number of statistical tests performed.

\section{SCZ-PRS}

The PRS comparing the European ancestry clinical sample with the independent control sample $(n=1528)$ has been reported previously. ${ }^{61}$ The PRSs are scaled to have a mean of 0 and a s.d. of 1 in a population sample. Briefly, and to illustrate the elevated psychiatric PRSs in the young clinical sample, the difference in mean SCZ-PRS between the clinical sample and controls was 0.54 control s.d. units $\left(P=1.6 \times 10^{-10}\right), 0.29$ control s.d. units for bipolar disorder $\left(P=5.1 \times 10^{-4}\right)$, and 0.46 control s.d. units for depression $\left(P=6.2 \times 10^{-8}\right)$. In contrast for the non-psychiatric traits of height, body mass index, coronary artery disease, type 2 diabetes and peptic ulcer disease, the differences between mean PRS for clinical participants and controls were non-significant.

In the following analyses, we focus only on the SCZ-PRS in those of European ancestry for three reasons. First, the GWAS used to generate the PRS is the largest. In terms of variance explained on the liability scale, the SCZ-PRS explains at least 7\%, whereas the respective PRSs for bipolar disorder and depression explain $4 \%$ of each disorder. ${ }^{8,62}$ Second, mental disorders are genetically correlated. For example, the genetic correlation between schizophrenia and bipolar disorder is high $(\sim 0.65),{ }^{12}$ and hence PRSs for these disorders will be correlated. Third, a high SCZ-PRS likely represents a genetic risk for mental disorders that is not specific for schizophrenia. Importantly, a high SCZ-PRS in the context of help-seeking young people could be useful in clinical decisionmaking. ${ }^{61}$

\section{Statistical analysis}

Analyses were performed using $\mathrm{R}$ statistical software with the RStudio IDE. ${ }^{63}$ Continuous data are summarised as means and $s$. d.s, and categorical data are summarised as frequencies and percentages. Linear regression was used for the continuous outcome and logistic regression for binary outcomes. As the SCZ-PRS is in s.d. units of the control group, odds ratios (OR) are interpretable as a 1 s.d. increase or decrease in the SCZ-PRS. Data were missing for $<5 \%$ of participants for each variable (Supplementary Table 1 available at https://doi.org/10.1192/bjo.2021.14) and all analyses were on 'complete cases'.

\section{Results}

\section{Participant characteristics}

Of the 2901 participants in the Optymise cohort, 193 patients were genotyped, and a total of 158 patients met all eligibility criteria. Characteristics of the final participants are in Table 1.

At baseline, the participants comprised 158 young people accessing youth mental health services; 99 were male $(62.7 \%)$ and 59 were female $(37.3 \%)$, with a mean age of 20.7 (s.d. $=4.7$ ) years at baseline (range 12-30). Around half the participants presented as stage $1 \mathrm{~b}$ $(53.8 \%, n=85)$ and around one-fifth presented as stage $2(20.9 \%$, $n=33$ ) (see Table 1 for clinical stage ratings). The majority of the participants presented with a primary mood (depressive or

\begin{tabular}{lc} 
Table 1 Baseline characteristics of 158 young people of European \\
ancestry presenting to mental health clinics & \multicolumn{1}{c}{ Values } \\
& $20.7(4.7)$ \\
Age, years: mean (s.d.) & $59(37.3)$ \\
Gender, female: $n$ (\%) & $58.4(10.2)$ \\
Social and Occupational Functioning Assessment Scale, & \\
$\quad$ mean (s.d.) & \\
Clinical stage, $n$ (\%) & $22(13.9)$ \\
1 1a - 'non-specific symptoms' & $85(53.8)$ \\
1 b - 'attenuated syndrome' & $33(20.9)$ \\
2 - 'discrete disorder' & $14(8.9)$ \\
3 - 'recurrent or persistent disorder' & $2(1.3)$ \\
4 - 'persistent, chronic and unremitting' & \\
Primary diagnosis, ${ }^{\text {b }} n$ (\%) & $15(9.5)$ \\
Anxiety disorder & $22(13.9)$ \\
Bipolar disorder & $60(38.0)$ \\
Depressive disorder & $26(16.5)$ \\
Psychotic disorder & $34(21.5)$ \\
Other & \\
a. Missing for $n=2$. & \\
b. Missing for $n=1$. &
\end{tabular}




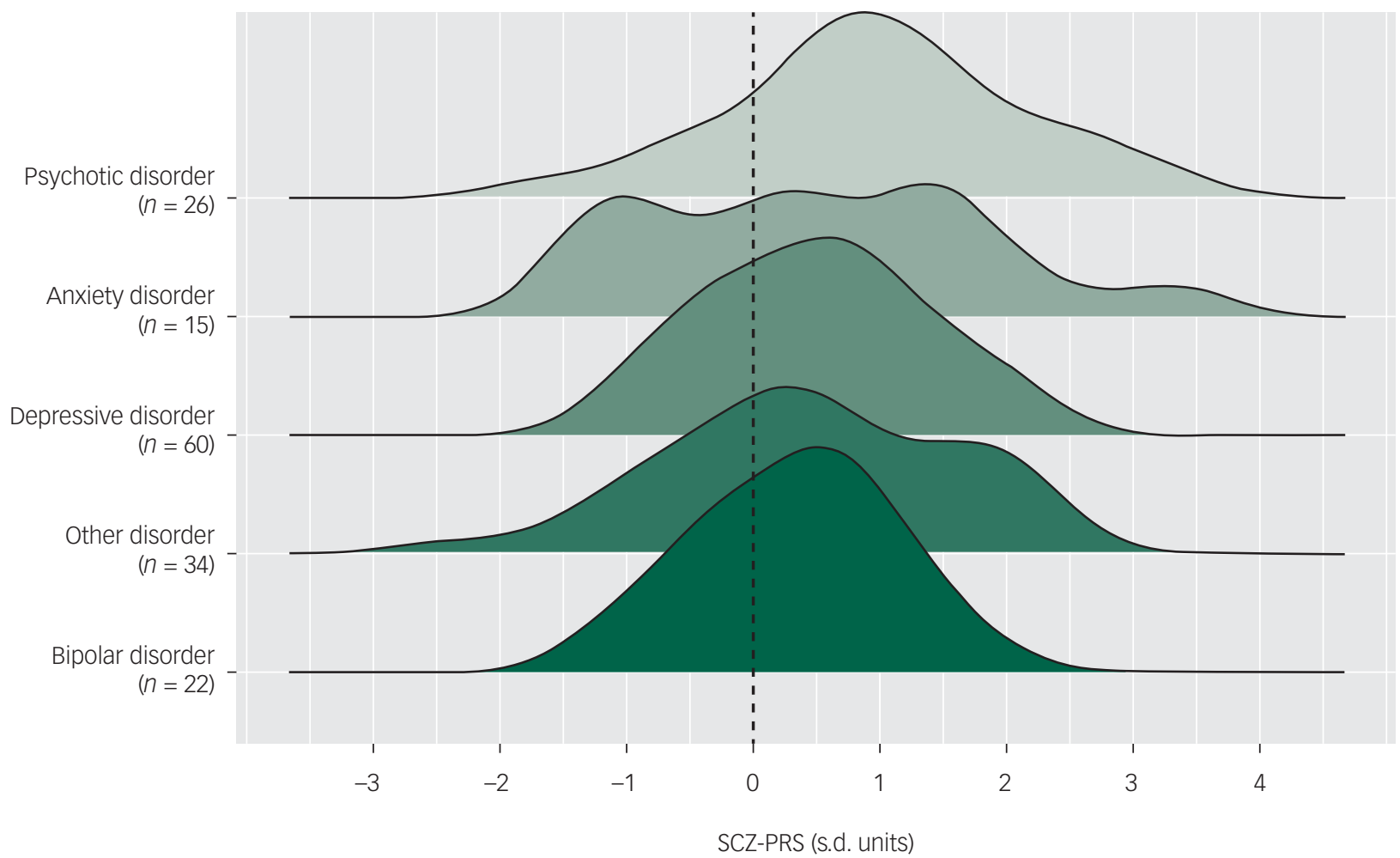

Fig. 1 Distributions of schizophrenia polygenic risk scores (SCZ-PRSs) by primary diagnoses at baseline, ordered by mean SCZ-PRSs (dashed line represents mean of control sample).

bipolar disorder) or anxiety syndrome $(61.4 \%, n=97)$ and around one-sixth presented with a primary psychotic syndrome $(16.5 \%$, $n=26)$.

Functional impairment was common, with a mean clinician rating of functioning on the SOFAS of 58.4 (s.d. = 10.2), falling in the band 'moderate difficulty in social, occupational or school functioning'. A total of 113 participants had longitudinal data (71.5\%), with a mean follow-up duration of 40.8 (s.d. $=30.5)$ months.

Compared with participants with only baseline data, those with follow-up data were on average younger $(20.0$ v. 22.5 years old; $P<0.002)$ and there was a trend toward more females $(42.5 \%$ v. $24.4 \% ; P=0.053)$. There was no difference in functioning as measured by the SOFAS (58.0 v. 59.7; $P=0.354$ ).

Numerical differences in proportions of diagnoses and clinical stages are shown in Supplementary Table 2.

\section{Associations between SCZ-PRS and diagnosis, clinical stage and functioning}

SCZ-PRS and diagnosis

At baseline, the pattern of SCZ-PRSs across primary diagnoses (i.e. the diagnosis identified as the main presenting problem) was highest among those with a psychotic disorder (mean 0.97, s.d. $=1.15, n=26$ ), followed by anxiety disorder (mean 0.51, s.d. $=1.35, n=15$ ), depressive disorder (mean 0.49, s.d. $=0.85$, $n=60$ ), other disorder (mean 0.45 , s.d. $=1.12, n=34$ ) and bipolar disorder (mean 0.32 , s.d. $=0.75, n=22$ ). The distributions of SCZPRS across disorders are shown in Fig. 1. Logistic regression showed that higher overall SCZ-PRS was associated with the presence of a primary psychotic disorder at baseline $(\mathrm{OR}=1.68$, 95\% CI 1.08-2.59, $P=0.020$ ).

Among the 113 participants with longitudinal data, five participants ( $\sim \%$ of the follow-up group) had a new-onset psychotic disorder (i.e. no psychotic disorder at baseline but incidence of psychotic disorder over follow-up). Participants who developed a new-onset psychotic disorder had numerically lower SCZ-PRSs (mean 0.28 , s.d. $=2.01$ ) than participants who had no psychotic disorder at baseline or over follow-up (mean 0.42 , s.d. $=0.95$ ). We did not observe a significant association between SCZ-PRS and new-onset psychotic disorder over follow-up $(\mathrm{OR}=0.84,95 \% \mathrm{CI}$ $0.34-2.03, P=0.69$ ).

\section{SCZ-PRS and clinical stage}

At baseline, the pattern of SCZ-PRSs was highest among those assigned stage $1 \mathrm{a}$ (mean 0.75 , s.d. $=0.91, n=22$ ), followed by stage 3 (mean 0.68, s.d. $=1.20, n=14$ ), stage $1 \mathrm{~b}$ (mean 0.52, s.d. $=1.04$, $n=85$ ), stage 2 (mean 0.38 , s.d. $=0.93, n=33$ ) and stage 4 (mean 0.34 , s.d. $=0.34, n=2$ ). Of note, the SCZ-PRS distributions were highly right-skewed for participants assigned stage 3, suggesting an overrepresentation of individuals with high SCZ-PRS scores (Fig. 2). However, a logistic regression did not show an association between overall SCZ-PRS and assignment at stage $2+$ at baseline $(\mathrm{OR}=0.90,95 \%$ CI $0.64-1.26, P=0.53)$.

Among those with follow-up data $(n=113)$, a total of 47 participants transitioned from a lower to a higher clinical stage over time in care. SCZ-PRSs were numerically similar among individuals who transitioned from a lower to a higher clinical stage (mean 0.44; s.d. $=1.01$ ) compared with those who did not transition (mean 0.46 ; s.d. $=1.03$ ). Logistic regression showed that the SCZ-PRS was not significantly associated with transition from a lower to a higher clinical stage $(\mathrm{OR}=1.02,95 \% \mathrm{CI} 0.70-1.48, P=0.92)$ or from a subthreshold clinical stage (1a or $1 \mathrm{~b}$ ) to a full-threshold (2+) clinical stage $(\mathrm{OR}=0.98,95 \%$ CI $0.66-1.44, P=0.90)$.

\section{SCZ-PRS and social and occupational functioning}

A linear regression showed that SCZ-PRS was not significantly associated with baseline functioning (SOFAS) $(R=0.03, P=0.76)$ 


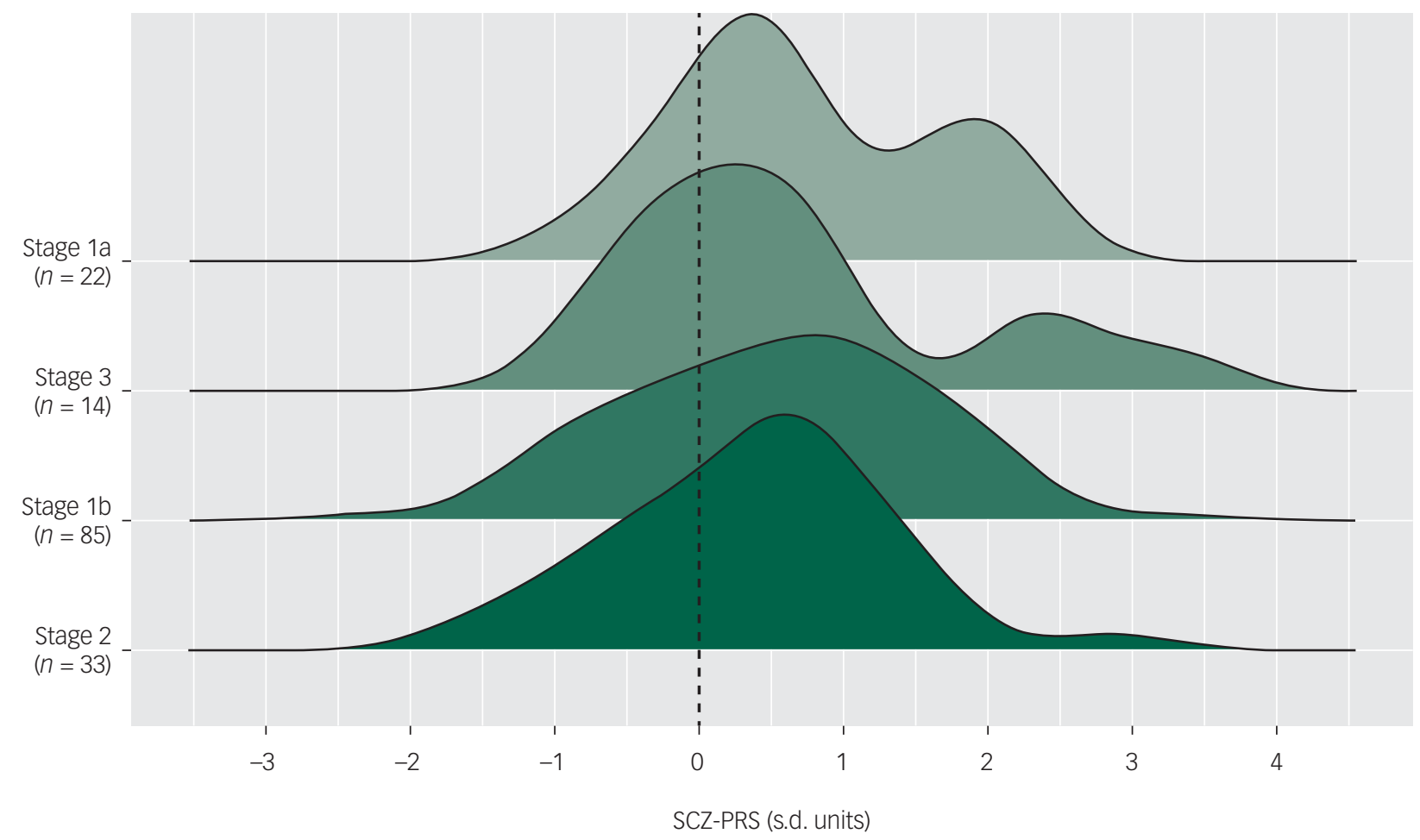

Fig. 2 Distribution of schizophrenia polygenic risk scores (SCZ-PRSs) by clinical stage at baseline, ordered by mean SCZ-PRSs (dashed line represents mean of control sample).

Stage 4 not displayed as $n<3$.

(Fig. 3). Among those with at least one follow-up time point, SCZPRS was also not significantly associated with being functionally impaired (i.e. SOFAS $<70)$ across two time points $(\mathrm{OR}=0.84$, 95\% CI 0.52-1.38, $P=0.50$ ).

\section{Exploratory analysis}

In an exploratory analysis, we examined the characteristics of participants who had a SCZ-PRS of $\geq 1.64$ (wherein $\sim 10 \%$ of the control participants are expected to fall) compared with a SCZ-PRS below 1.64. We show in Table 2 that there were numerically higher proportions of participants in the 'high' SCZ-PRS group $(\geq 1.64)$ compared with the 'low' SCZ-PRS group $(<1.64)$ who had a psychotic disorder at baseline $(22.7 \% v .15 .4 \%)$ or a new-onset psychotic disorder over follow-up (7.1\% v. 4.0\%).

\section{Discussion}

\section{Principal findings}

To our knowledge, this is the first study to examine the clinical utility of the SCZ-PRS in a transdiagnostic clinical cohort of adolescents and young adults in the early phases of mental disorders. We observed an association between higher overall SCZ-PRS and diagnosis of a psychotic disorder at baseline, supporting the link between the SCZ-PRS and liability towards psychotic disorders such as schizophrenia. ${ }^{18}$ We did not find evidence of a significant relationship between the SCZ-PRS and functioning or assignment at clinical stage $2+$ at baseline. Although underpowered, our longitudinal analyses did not detect associations between the SCZ-PRS and an impaired course of functioning, nor for incidence of psychotic disorder or clinical stage transition over follow-up. However, these non-significant findings are not surprising given the patterns of associations at baseline. Of note, SCZ-PRS were higher in all patient diagnostic groups compared to the control participants, consistent with recent findings of pleiotropic effects of the SCZPRS on other non-psychotic mental disorders. ${ }^{18}$

\section{SCZ-PRSs and psychotic disorder}

Numerous studies to date have demonstrated an association between the SCZ-PRS and schizophrenia, ${ }^{2,64,65}$ with some also reporting associations with a broader spectrum of disorders including schizoaffective disorder, psychotic disorder not otherwise specified and bipolar I disorder. ${ }^{64,66}$ Notably, the OR of SCZ-PRSs on presence of a psychotic disorder at baseline in the current study $(\mathrm{OR}=1.68)$ is similar to that reported for SCZ-PRSs and schizophrenia in a recent large study across four US healthcare systems $(\mathrm{OR}=1.55) .{ }^{18}$ A recent study examined whether the SCZ-PRS can be used to predict incident psychotic disorders, reporting modest improvement of prediction of conversion to schizophrenia with the addition of the SCZ-PRS to an existing risk calculator among individuals at clinical high risk of schizophrenia. ${ }^{19}$ Although we did not observe a significant association between the SCZ-PRS and incidence of a new-onset psychotic disorder over follow-up, larger studies will be needed to more conclusively determine whether this type of prediction has utility beyond high-risk cohorts.

\section{SCZ-PRS and social and occupational functioning}

As the SCZ-PRS has been reported to be associated with severity of neurocognitive impairment, ${ }^{22,23}$ negative symptoms ${ }^{24}$ and poorer course of illness defined by the Global Assessment of Functioning, ${ }^{23}$ we speculated that the SCZ-PRS might be related to functional impairment. However, our findings did not offer support for such a relationship. 


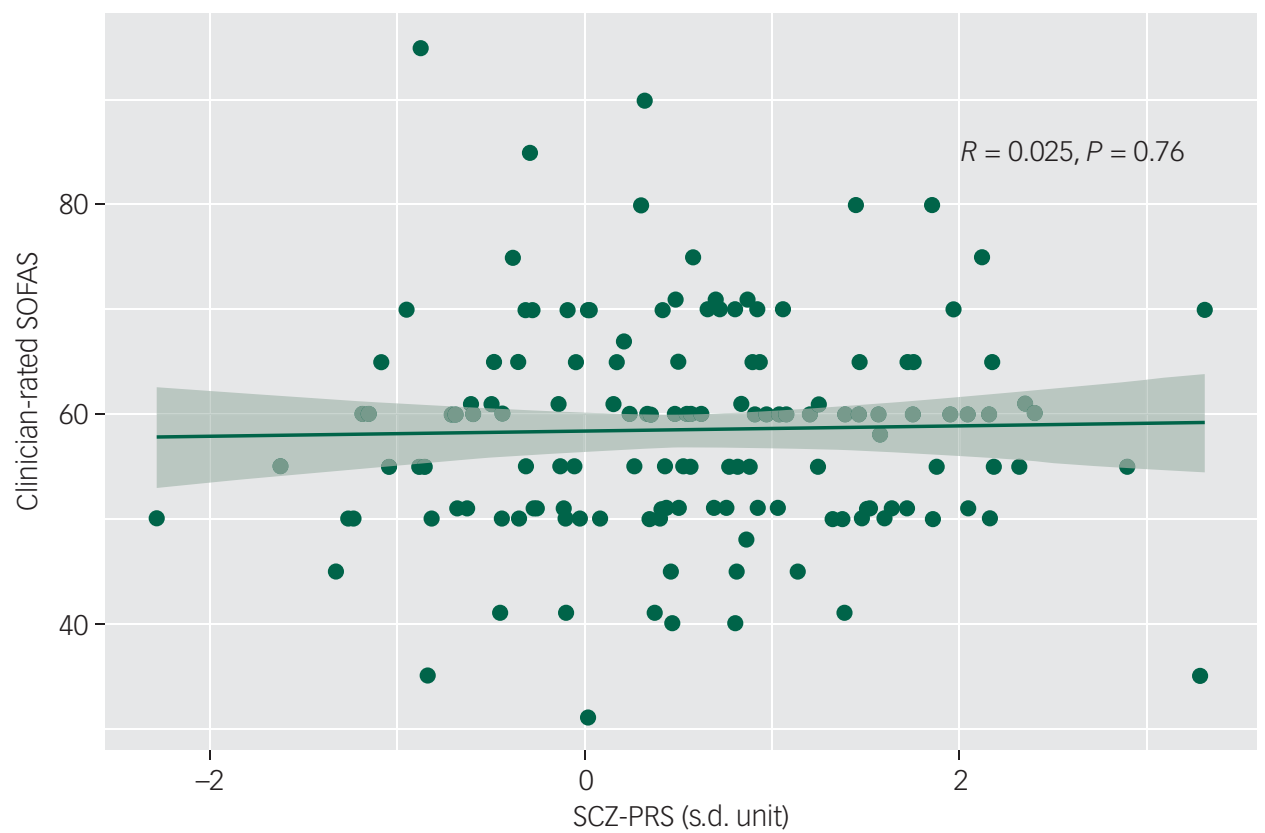

Fig. 3 Association of schizophrenia polygenic risk score (SCZ-PRS) and baseline social and occupational functioning among young people accessing mental health services

SOFAS, Social and Occupational Functioning Assessment Scale.

\section{SCZ-PRS and clinical stage}

Several studies have suggested that the SCZ-PRS is associated with a poorer course of illness, namely chronic ${ }^{25}$ and treatment-resistant schizophrenia. ${ }^{67-69}$ Accordingly, we wondered whether the SCZPRS might also be associated with a more severe illness course transdiagnostically, as determined by greater stage of illness in a transdiagnostic clinical staging model. ${ }^{32,33,70}$ However, our analyses did not show an association for assignment at clinical stage $2+$ at baseline. Similarly, the SCZ-PRS was not significantly associated with transition to a more advanced clinical stage over follow-up.

Two qualifying points are worth noting. First, of the 49 participants who were assigned stage $2+$ at baseline, only around one-third had a psychotic disorder $(n=19,38.8 \%)$. Second, of the 38 participants that transitioned to a stage $2+$ disorder over follow-up, almost three-quarters transitioned to non-psychotic disorders $(71.1 \%$, $n=27$ ). Two potential interpretations of these findings are that the SCZ-PRS may not be associated with transition to more advanced stages of non-psychotic disorders, or alternatively, the SCZ-PRS may not be robustly associated with course of psychotic disorders more specifically, as suggested by some studies. ${ }^{26,27}$ Larger clinical studies with greater statistical power will be needed to clarify these points.

Table 2 Key characteristics of individuals with a 'high' schizophrenia polygenic risk score (SCZ-PRS, 21.64) and 'low' SCZ-PRS $(<1.64)$

\begin{tabular}{lcc|} 
& 'High' $(\geq 1.64)$ & 'Low' $(<1.64)$ \\
& SCZ-PRS & SCZ-PRS \\
& $(n=22)$ & $(n=136)$ \\
\hline Age, years: & $20.1(3.7)$ & $20.8(4.8)$ \\
Gender, female: $n$ (\%) & $6(27.3)$ & $53(39.0)$ \\
Baseline psychotic disorder, $n$ (\%) & $5(22.7)$ & $21(15.4)^{\mathrm{a}}$ \\
New-onset psychotic disorder, ${ }^{\text {b }} n(\%)$ & $1(7.1)$ & $4(4.0)^{\mathrm{c}}$ \\
Clinical stage transition, ${ }^{\mathrm{b}} n(\%)$ & $4(28.6)$ & $43(43.4)^{\mathrm{c}}$ \\
$\begin{array}{l}\text { a. Missing for } n=1 \text {. } \\
\text { b. Participants with follow-up data: } n=113 .\end{array}$ & & \\
c. Missing for $n=1$. & & \\
\hline
\end{tabular}

\section{Limitations}

Several limitations should be mentioned. First, the SCZ-PRS were derived from a European ancestry discovery sample, and these scores have been reported to have poorer accuracy in nonEuropean ancestries. ${ }^{71}$ Although we focused our main analyses on participants with European ancestry, it is worth noting that the East Asian ancestry group had substantially higher SCZ-PRS scores (Supplementary Fig. 1). Speculatively, there may be important cultural factors influencing thresholds for help-seeking in this East Asian ancestry group. For example, some individuals may 'require' very severe illness (and possibly high polygenic burden) in order to cross thresholds for seeking care. Importantly, all of our findings were robust to sensitivity analyses including European, East Asian and other ancestries. Nonetheless, ancestrally diverse GWASs are critical to achieve more generalisable and equitable PRSs. ${ }^{71}$

Second, the SCZ-PRS reflects variation captured by individual SNPs of small effect and does not capture rare SNPs or de novo mutations of larger effect (such as copy number variants, deletions). Third, and critically, the SCZ-PRS used in this study captures 7\% of the genetic liability to schizophrenia, and as such, larger GWAS studies are needed to increase the predictive power of the SCZ-PRS in clinical contexts.

Fourth, for reasons related to sample size, we focused our analyses on the overall SCZ-PRS (analysed as a continuous variable). A recent editorial ${ }^{61}$ has, however, suggested that an optimal use of the SCZ-PRS may be to focus prediction efforts on a select subgroup with high SCZ-PRSs (as in our exploratory analysis; see Table 2), for whom this information may influence clinical decisionmaking. Larger clinical studies with higher statistical power are needed to better understand these relationships.

Fifth, the subset of the cohort who were genotyped were not randomly selected, and our results may not be fully generalisable to the broader help-seeking populations accessing transdiagnostic youth mental health services. Relatedly, data regarding the quantity, quality, and intensity of treatment and engagement was not 
systematically captured, and it is possible that heterogeneity in treatment patterns may confound some of our findings. Finally, incidence of psychotic disorder over follow-up was a relatively rare event ( $n=5 ; \sim 4 \%$ longitudinal sample) and larger studies are needed to better answer the question of whether the SCZ-PRS can predict new cases and tilt clinical decision-making. ${ }^{72}$

\section{Future directions}

The SCZ-PRS will very likely be improved in coming years with the addition of novel SNPs identified in larger GWASs and may be further strengthened by inclusion of rarer genetic variants with larger effects. Critically, these developments may improve the predictive power of the SCZ-PRS and support its inclusion in clinical decision-making. Larger clinical studies focusing on subgroups with high SCZ-PRSs will be crucial for testing this hypothesis.

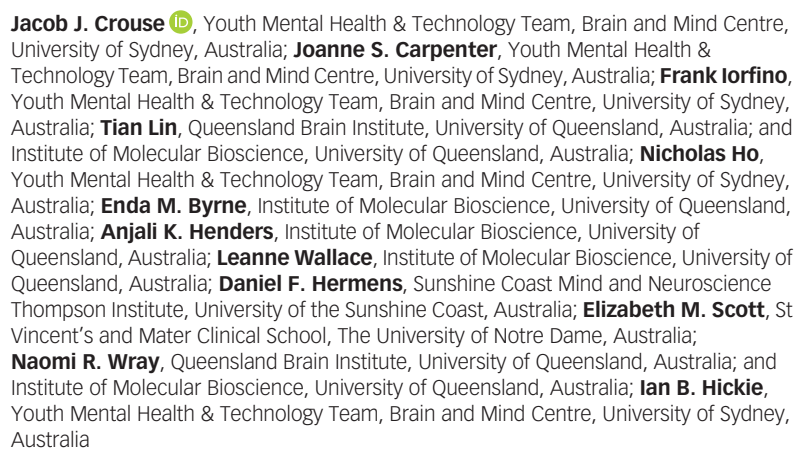

\section{Supplementary material}

Supplementary material is available online at https://doi.org/10.1192/bjo.2021.14

\section{Data availability}

The data that support the findings of this study are available on reasonable request from the The data that support the findings of this study are available on reasonable request from the
corresponding author. J.J.C. The data are not publicly available due to their containing informacorresponding author, J.J.C. The data are not publicly available due to
tion that could compromise the privacy of research participants.

\section{Acknowledgements}

The authors gratefully thank all of the young people who participated in this study

\section{Author contributions}

J.J.C. analysed the data and wrote the manuscript. J.J.C., J.S.C., F.I., T.L., E.M.B., A.K.H., L.W. D.F.H., E.M.S., N.H., N.R.W. and I.B.H. collected and processed the data. All authors contributed D.F.H., E.M.S., N.H., N.R.W. and I.B.H. collected
intellectually and revised the manuscript.

\section{Funding}

This study was supported by grants from the National Health \& Medical Research Council (NHMRC) including: Centre of Research Excellence (I.B.H., 1061043), Fellowship (I.B.H., 1046899). Clinical Research Fellowship (I.B.H. 402864), Program Grant (N.R.W. 1113400) and Investigator Grant (N.R.W., 1173790). The funders had no role in study design, data collection and analysis, decision to publish or preparation of the manuscript.

\section{Declaration of interest}

E.M.S. reports personal fees from St Vincent's Private Hospital, grants from Servier, personal fees from Servier, personal fees from Eli-Lilly, personal fees from Pfizer, outside the submitted work. She has received honoraria for educational seminars related to the clinical management of depressive disorders supported by Servier and Eli-Lilly pharmaceuticals. She has participated in a national advisory board for the antidepressant compound Pristiq, manufactured by Pfizer. She was the National Coordinator of an antidepressant trial sponsored by Servier. I.B.H reports personal fees from the National Mental Health Commission, personal fees from Medibank Clinical Reference Group, non-financial support from Psychosis Australia Trust, grants from NHMRC, grants from Innowell Pty LTD, grants from NHMRC, grants from NHMRC, outside the submitted work I BH was an inaugural Commissioner on Australia's National Mental Health Commission (2012-18). He is the Co-Director, Health and Policy at the Brain and Mind Centre
(BMC) University of Sydney. The BMC operates an early-intervention youth services at Camperdown under contract to headspace. I.B.H. has previously led community-based and pharmaceutical industry-supported (Myeth, Eli Lily, Servier, Pfizer, Astrazeneca) projects focused on the identification and better management of anxiety and depression. He was a member of the Medical Advisory Panel for Medibank Private until October 2017, a Board Member of Psychosis Australia Trust and a member of Veterans Mental Health Clinical Reference group. He is the Chief Scientific Advisor to, and an equity shareholder in Innowell. Innowell has been formed by the University of Sydney and PwC to deliver the $\$ 30$ m Australian Government-funded 'Project Synergy'. Project Synergy is a 3-year programme J.J.C., J.S.C., F.I., T.L., N.H., E.M.B., A.K.H., L.W., D.F.H. and N.R.W. report no competing interests.

\section{References}

1 MacDonald ME, Ambrose CM, Duyao MP, Myers RH, Lin C, Srinidhi L, et al. A novel gene containing a trinucleotide repeat that is expanded and unstable on Huntington's disease chromosomes. Cell 1993; 72: 971-83.

2 Schizophrenia Working Group of the Psychiatric Genomics Consortium. Biological insights from 108 schizophrenia-associated genetic loci. Nature 2014; 511: 421-7

3 International Schizophrenia Consortium. Rare chromosomal deletions and duplications increase risk of schizophrenia. Nature 2008; 455: 237-41.

4 Purcell SM, Wray NR, Stone JL, Visscher PM, O'Donovan MC, Sullivan PF, et al. Common polygenic variation contributes to risk of schizophrenia and bipolar disorder. Nature 2009; 460: 748-52.

5 Lee J-M, Wheeler Vanessa C, Chao Michael J, Vonsattel Jean Paul G, Pinto Ricardo $\mathrm{M}$, Lucente $\mathrm{D}$, et al. Identification of genetic factors that modify clinical onset of Huntington's disease. Cell 2015; 162: 516-26.

6 Kuchenbaecker KB, McGuffog L, Barrowdale D, Lee A, Soucy P, Dennis J, et al. Evaluation of polygenic risk scores for breast and ovarian cancer risk prediction in BRCA1 and BRCA2 mutation carriers. J Natl Cancer Inst 2017; 109: djw302.

7 Wray NR, Ripke S, Mattheisen M, Trzaskowski M, Byrne EM, Abdellaoui A, et al. Genome-wide association analyses identify 44 risk variants and refine the genetic architecture of major depression. Nat Genet 2018; 50: 668-81.

8 Stahl EA, Breen G, Forstner AJ, McQuillin A, Ripke S, Trubetskoy V, et al. Genome-wide association study identifies 30 loci associated with bipolar disorder. Nat Genet 2019; 51: 793-803.

9 Demontis D, Walters RK, Martin J, Mattheisen M, Als TD, Agerbo E, et al. Discovery of the first genome-wide significant risk loci for attention deficit/ hyperactivity disorder. Nat Genet 2019; 51: 63-75.

10 Levey DF, Gelernter J, Polimanti R, Zhou H, Cheng Z, Aslan M, et al. Reproducible genetic risk loci for anxiety: results from approximately 200,000 participants in the Million Veteran Program. Am J Psychiatry 2020; 177: 223-32.

11 Howard DM, Adams MJ, Clarke T-K, Hafferty JD, Gibson J, Shirali M, et al. Genome-wide meta-analysis of depression identifies 102 independent variants and highlights the importance of the prefrontal brain regions. Nat Neurosci 2019; 22: 343-52.

12 Lee SH, Ripke S, Neale BM, Faraone SV, Purcell SM, Perlis RH, et al. Genetic relationship between five psychiatric disorders estimated from genome-wide SNPs. Nat Genet 2013; 45: 984-94.

13 Anttila V, Bulik-Sullivan B, Finucane $\mathrm{HK}$, Walters RK, Bras J, Duncan L, et al. Analysis of shared heritability in common disorders of the brain. Science 2018; 360: eaap8757

14 Lee PH, Anttila V, Won H, Feng Y-CA, Rosenthal J, Zhu Z, et al. Genomic relationships, novel loci, and pleiotropic mechanisms across eight psychiatric disorders. Cell 2019; 179: 1469-82.e11.

15 Network and Pathway Analysis Subgroup of Psychiatric Genomics Consortium. Psychiatric genome-wide association study analyses implicate neuronal, immune and histone pathways. Nat Neurosci 2015; 18: 199-209.

16 Murray GK, Lin T, Austin J, McGrath JJ, Hickie IB, Wray NR. Could polygenic risk scores be useful in psychiatry?: a review. JAMA Psychiatry [Epub ahead of print] 14 Oct 2020. Available from: https://doi.org/10.1001/jamapsychiatry.2020. 3042.

17 Wray NR, Lee SH, Mehta D, Vinkhuyzen AA, Dudbridge F, Middeldorp CM. Research review: polygenic methods and their application to psychiatric traits. J Child Psychol Psychiatry 2014; 55: 1068-87.

18 Zheutlin AB, Dennis J, Karlsson Linner R, Moscati A, Restrepo N, Straub P, et al. Penetrance and pleiotropy of polygenic risk scores for schizophrenia in 106,160 patients across four health care systems. Am J Psychiatry 2019; 176: $846-55$.

19 Perkins DO, Olde Loohuis L, Barbee J, Ford J, Jeffries CD, Addington J, et al. Polygenic risk score contribution to psychosis prediction in a target population of persons at clinical high risk. Am J Psychiatry 2020; 177: 155-63. 
20 Neilson E, Bois C, Clarke TK, Hall L, Johnstone EC, Owens DGC, et al. Polygenic risk for schizophrenia, transition and cortical gyrification: a high-risk study. Psychol Med 2018; 48: 1532-9.

21 Musliner KL, Krebs MD, Albiñana C, Vilhjalmsson B, Agerbo E, Zandi PP, et al. Polygenic risk and progression to bipolar or psychotic disorders among individuals diagnosed with unipolar depression in early life. Am J Psychiatry 2020; 177: 936-43.

22 Germine L, Robinson EB, Smoller JW, Calkins ME, Moore TM, Hakonarson H et al. Association between polygenic risk for schizophrenia, neurocognition and social cognition across development. Transl Psychiatry 2016; 6: e924.

23 Jonas KG, Lencz T, Li K, Malhotra AK, Perlman G, Fochtmann LJ, et al. Schizophrenia polygenic risk score and 20-year course of illness in psychotic disorders. Transl Psychiatry 2019; 9: 300

24 Fanous AH, Zhou B, Aggen SH, Bergen SE, Amdur RL, Duan J, et al. Genomewide association study of clinical dimensions of schizophrenia: polygenic effect on disorganized symptoms. Am J Psychiatry 2012; 169: 1309-17.

25 Meier SM, Agerbo E, Maier R, Pedersen CB, Lang M, Grove J, et al. High loading of polygenic risk in cases with chronic schizophrenia. Mol Psychiatry 2016; 21: 969-74.

26 Wimberley T, Gasse C, Meier SM, Agerbo E, MacCabe JH, Horsdal HT. Polygenic risk score for schizophrenia and treatment-resistant schizophrenia. Schizophr Bull 2017; 43: 1064-9.

27 Legge SE, Dennison CA, Pardiñas AF, Rees E, Lynham AJ, Hopkins L, et al. Clinical indicators of treatment-resistant psychosis. Br J Psychiatry 2020; 216: 259-66.

28 Richards AL, Pardiñas AF, Frizzati A, Tansey KE, Lynham AJ, Holmans P, et al. The relationship between polygenic risk scores and cognition in schizophrenia. Schizophr Bull 2020; 46: 336-44.

29 Shafee $\mathrm{R}$, Nanda $\mathrm{P}$, Padmanabhan JL, Tandon N, Alliey-Rodriguez $\mathrm{N}$ Kalapurakkel S, et al. Polygenic risk for schizophrenia and measured domains of cognition in individuals with psychosis and controls. Transl Psychiatry 2018; 8: 78

30 Xavier RM, Dungan JR, Keefe RSE, Vorderstrasse A. Polygenic signal for symptom dimensions and cognitive performance in patients with chronic schizophrenia. Schizophr Res 2018; 12: 11-9.

31 Musliner KL, Mortensen PB, McGrath JJ, Suppli NP, Hougaard DM, BybjergGrauholm J, et al. Association of polygenic liabilities for major depression, bipolar disorder, and schizophrenia with risk for depression in the danish population. JAMA Psychiatry 2019; 76: 516-25.

32 McGorry $\mathrm{P}$, Keshavan $\mathrm{M}$, Goldstone $\mathrm{S}$, Amminger $\mathrm{P}$, Allott $\mathrm{K}$, Berk $\mathrm{M}$, et al Biomarkers and clinical staging in psychiatry. World Psychiatry 2014; 13: 211-23.

33 Shah JL, Scott J, McGorry PD, Cross SPM, Keshavan MS, Nelson B, et al Transdiagnostic clinical staging in youth mental health: a first international consensus statement. World Psychiatry 2020; 19: 233-42.

34 McGorry PD, Hartmann JA, Spooner R, Nelson B. Beyond the "at risk mental state" concept: transitioning to transdiagnostic psychiatry. World Psychiatry 2018; 17: 133-42.

35 Dalgleish T, Black M, Johnston D, Bevan A. Transdiagnostic approaches to mental health problems: current status and future directions. J Consult Clin Psychol 2020; 88: 179-95.

36 Goodkind $\mathrm{M}$, Eickhoff SB, Oathes DJ, Jiang Y, Chang A, Jones-Hagata LB, et al Identification of a common neurobiological substrate for mental illness. JAMA Psychiatry 2015; 72: 305-15.

37 Hyman SE. The diagnosis of mental disorders: the problem of reification. Annu Rev Clin Psychol 2010; 6: 155-79.

38 Krueger RF, Eaton NR. Transdiagnostic factors of mental disorders. World Psychiatry 2015; 14: 27-9.

39 Crouse JJ, Chitty KM, Iorfino F, Carpenter JS, White D, Nichles A, et al. Transdiagnostic neurocognitive subgroups and functional course in young people with emerging mental disorders: a cohort study. BJPsych Open 2020; 6: e31.

40 McGorry PD, Goldstone SD, Parker AG, Rickwood DJ, Hickie IB. Cultures for mental health care of young people: an Australian blueprint for reform. Lancet Psychiatry 2014; 1: 559-68.

41 van Os J. The dynamics of subthreshold psychopathology: implications for diag nosis and treatment. Am J Psychiatry 2013; 170: 695-8.

42 Wittchen $\mathrm{H}-\mathrm{U}$, Lieb R, Pfister $\mathrm{H}$, Schuster $\mathrm{P}$. The waxing and waning of mental disorders: evaluating the stability of syndromes of mental disorders in the population. Compr Psychiatry 2000; 41 (2, suppl. 1): 122-32.

43 Ev E, Altman DG, Egger M, Pocock SJ, Gøtzsche PC, Vandenbroucke JP. Strengthening the reporting of observational studies in epidemiology (STROBE) statement: guidelines for reporting observational studies. BMJ 2007; 335: 806-8

44 Carpenter JS, Iorfino F, Cross S, Nichles A, Zmicerevska N, Crouse J, et al. Cohort profile: the Brain and Mind Centre Optymise cohort: tracking multidimensional outcomes in young people presenting for mental healthcare. BMJ Open 2020; 10: e030985.

45 Iorfino F, Scott EM, Carpenter JS, Cross SP, Hermens DF, Killedar M, et al. Clinical stage transitions in persons aged 12 to 25 years presenting to early intervention mental health services with anxiety, mood, and psychotic disorders. JAMA Psychiatry 2020; 76: 1167-75.

46 Crouse JJ, Chitty KM, Iorfino F, Carpenter JS, White D, Nichles A, et al. Modelling associations between neurocognition and functional course in young people with emerging mental disorders: a Iongitudinal cohort study. Trans Psychiatry 2020; 10: 22

47 Crouse JJ, Chitty KM, Iorfino F, White D, Nichles A, Zmicerevska N, et al Exploring associations between early substance use and longitudinal sociooccupational functioning in young people engaged in a mental health service. PLOS One 2019; 14: e0210877.

48 American Psychiatric Association. Diagnostic and Statistical Manual of Mental Disorders (5th edn). APA, 2013.

49 Hickie IB, Scott EM, Hermens DF, Naismith SL, Guastella AJ, Kaur M, et al. Applying clinical staging to young people who present for mental health care. Early Interv Psychiatry 2013; 7: 31-43.

50 MCGorry PD, Hickie IB, Yung AR, Pantelis C, Jackson HJ. Clinical staging of psychiatric disorders: a heuristic framework for choosing earlier, safer and more effective interventions. Aust N Z J Psychiatry 2006; 40: 616-22.

51 Goldman $\mathrm{HH}$, Skodol AE, Lave TR. Revising Axis V for DSM-IV: A Review of Measures of Social Functioning. Am J Psychiatry 1992; 149(9): 1148-56.

52 Rickwood DJ, Mazzer KR, Telford NR, Parker AG, Tanti CJ, McGorry PD. Changes in psychological distress and psychosocial functioning in young people visiting headspace centres for mental health problems. Med J Aust 2015; 202: 537-42.

53 Nalls MA, Blauwendraat C, Vallerga CL, Heilbron K, Bandres-Ciga S, Chang D, et al. Identification of novel risk loci, causal insights, and heritable risk for Parkinson's disease: a meta-analysis of genome-wide association studies. Lancet Neurol 2019; 18: 1091-102.

54 The Haplotype Reference Consortium. A reference panel of 64,976 haplotypes for genotype imputation. Nat Genet 2016; 48: 1279-83.

55 Abecasis GR, Altshuler D, Auton A, Brooks LD, Durbin RM, Gibbs RA, et al. A map of human genome variation from population-scale sequencing. Nature 2010; 467: 1061-73.

56 Lloyd-Jones LR, Zeng J, Sidorenko J, Yengo L, Moser G, Kemper KE, et al. Improved polygenic prediction by Bayesian multiple regression on summary statistics. Nat Commun 2019; 10: 5086

57 Pardiñas AF, Holmans P, Pocklington AJ, Escott-Price V, Ripke S, Carrera N, et al. Common schizophrenia alleles are enriched in mutation-intolerant genes and in regions under strong background selection. Nat Genet 2018; 50: 381-9.

58 Yengo L, Sidorenko J, Kemper KE, Zheng Z, Wood AR, Weedon MN, et al. Metaanalysis of genome-wide association studies for height and body mass index in approximately 700000 individuals of European ancestry. Hum Mol Genet 2018. 27: 3641-9.

59 Xue A, Wu Y, Zhu Z, Zhang F, Kemper KE, Zheng Z, et al. Genome-wide association analyses identify 143 risk variants and putative regulatory mechanisms for type 2 diabetes. Nat Commun 2018; 9: 2941.

60 Wu Y, Murray GK, Byrne EM, Sidorenko J, Visscher PM, Wray NR. Genomewide association study of gastrointestinal disorders reinforces the link between the digestive tract and the nervous system. BioRxiv [Preprint] 2019. Available from: https://www.biorxiv.org/content/10.1101/811737v1.

61 Wray NR, Lin T, Austin J, McGrath JJ, Hickie IB, Murray GK, et al. From basic science to clinical application of polygenic risk scores: a primer. JAMA Psychiatry 2021; 78: 101-9.

62 Ni G, Zeng J, Revez JA, Wang Y, Ge T, Restuadi R, et al. A comprehensive evaluation of polygenic score methods across cohorts in psychiatric disorders. medRxiv [Preprint] 2020. Available from: https://www.medrxiv.org/ content/10.1101/2020.09.10.20192310v1.

63 R Development Team. R: A language and environment for statistical computing. R Foundation for Statistical Computing, 2010.

64 Tesli M, Espeseth T, Bettella F, Mattingsdal M, Aas M, Melle I, et al. Polygenic risk score and the psychosis continuum model. Acta Psychiatr Scand 2014; 130: 311-7.

65 Agerbo E, Sullivan PF, Vilhjálmsson BJ, Pedersen CB, Mors O, Børglum AD, et al. Polygenic risk score, parental socioeconomic status, family history of psychiatric disorders, and the risk for schizophrenia: a danish population-based study and meta-analysis. JAMA Psychiatry 2015; 72: 635-41.

66 Calafato MS, Thygesen JH, Ranlund S, Zartaloudi E, Cahn W, Crespo-Facorro B, et al. Use of schizophrenia and bipolar disorder polygenic risk scores to identify psychotic disorders. Br J Psychiatry 2018; 213: 535-41.

67 Gasse C, Wimberley T, Wang Y, Mors O, Borglum A, Als TD, et al. Schizophrenia polygenic risk scores, urbanicity and treatment-resistant schizophrenia. Schizophr Res 2019; 212: 79-85. 
68 Zhang JP, Robinson D, Yu J, Gallego J, Fleischhacker WW, Kahn RS, et al. Schizophrenia polygenic risk score as a predictor of antipsychotic efficacy in first-episode psychosis. Am J Psychiatry 2019; 176: 21-8.

69 Werner MCF, Wirgenes KV, Haram M, Bettella F, Lunding SH, Rødevand L, et al. Indicated association between polygenic risk score and treatment-resistance in a naturalistic sample of patients with schizophrenia spectrum disorders. Schizophr Res 2020; 218: 55-62.

70 Hickie IB, Scott EM, Cross SP, Iorfino F, Davenport TA, Guastella AJ, et al. Right care, first time: a highly personalised and measurement-based care model to manage youth mental health. Med J Aust 2019; 211 (suppl. 9): S3-S46.
71 Martin AR, Kanai M, Kamatani Y, Okada Y, Neale BM, Daly MJ. Clinical use of current polygenic risk scores may exacerbate health disparities. Nat Genet 2019; 51: 584-91.

72 Rohleder C, Song YJC, Crouse JJ, Davenport TA, Iorfino F, Hamilton B, et al. Youth Mental Health Tracker: protocol to establish a longitudinal cohort and research database for young people attending Australian mental health services. BMJ Open 2020; 10: e035379. 